\title{
RELAÇÃO DA MORFOMETRIA E COMPETIÇÃO COM O CRESCIMENTO DE Trichilia claussenii EM UM FRAGMENTO DE FLORESTA SEMIDECIDUAL, RS
}

\author{
Bruna Denardin da Silveira ${ }^{1}$, Eduardo Pagel Floriano ${ }^{2}$, Nelson Yoshihiro Nakajima $^{3}$, \\ Roberto Tuyoshi Hosokawa ${ }^{3}$, Nelson Carlos Rosot $^{3}$, Cibele Rosa Gracioli ${ }^{2}$ \\ ${ }^{1}$ Universidade Federal do Paraná, Programa de Pós-Graduação em Engenharia Florestal, Curitiba, Paraná, Brasil - \\ brunadenardin@unipampa.edu.br \\ ${ }^{2}$ Universidade Federal do Pampa, Campus São Gabriel, Rio Grande do Sul, Brasil - eduardofloriano@unipampa.edu.br; \\ cibelegracioli@unipampa.edu.br \\ ${ }^{3}$ Universidade Federal do Paraná, Departamento de Ciências Florestais, Curitiba, Paraná, Brasil - nelson.nakajima @ufpr.br; \\ rth@japan.org.br; ncrosot@ufpr.br
}

Recebido para publicação: 29/11/2012 - Aceito para publicação: 24/09/2014

\begin{abstract}
Resumo
Este trabalho teve como objetivos determinar a relação de variáveis morfométricas e de índices de competição com o crescimento em diâmetro de Trichilia claussenii, em um fragmento da Floresta Estacional Semidecidual na região central do Rio Grande do Sul. Os dados utilizados neste estudo foram obtidos de 29 árvores amostradas por classe diamétrica, denominadas árvores centrais, com diâmetro à altura do peito (d) igual ou superior a 10,0 centímetros, distribuídas em 51 parcelas permanentes, totalizando 0,51 hectares. Cada árvore central teve as seguintes variáveis mensuradas: altura total e altura comercial do fuste, diâmetro à altura do peito, diâmetro da copa e comprimento de copa. Posteriormente, calcularam-se alguns parâmetros morfométricos, assim como alguns índices de competição dependentes e independentes da distância. A análise de correlação de Pearson e análise de regressão foram utilizadas para verificar a relação entre as variáveis morfométricas, os índices de competição e o incremento em diâmetro. Concluiu-se que existe associação entre o incremento em diâmetro de Trichilia claussenii e a competição, sendo que o crescimento em diâmetro possui maior relação com o índice Bal de competição.

Palavras-chave: Catiguá; floresta nativa; biometria florestal.
\end{abstract}

\begin{abstract}
Relationship of morphometry and competition with growth of Trichilia claussenii in fragment of Semidecidual Forest, RS. This study aimed to determine the relationship of morphometric variables and indices of competition with the growth in diameter of Trichilia claussenii, in a fragment of semideciduous forest in the central region of Rio Grande do Sul. The data used in this study were obtained from 29 sampled trees by diameter class, called central trees with diameter at breast height (d) not less than $10.0 \mathrm{~cm}$, from 51 permanent plots, totaling 0.51 ha. Each central tree had measured the following variables: total height and commercial height, stem diameter at breast height, crown diameter and crown length. Subsequently, it was calculated some morphometric parameters, as well as some competition indexes dependent and independent of distance. The Pearson correlation analysis and regression analysis were used to verify the relationship between the morphometric variables, competition indexes and diameter increment. It was concluded that there is an association between the increase in diameter of Trichilia claussenii and competition, where the growth in diameter has a greater relationship with $\mathrm{Bal}$ index of competition.

Keywords: Catigua; native forest; forest biometrics.
\end{abstract}

\section{INTRODUÇÃO}

Na região central do estado do Rio Grande do Sul, uma grande proporção de floresta nativa sofreu processo de alteração antrópica, em consequência do uso agrícola e desenvolvimento urbano, descaracterizando sua estrutura natural (LONGHI, 2000). Por isso, é evidente a relativa escassez ou até

FLORESTA, Curitiba, PR, v. 45, n. 2, p. 373 - 382, abr. / jun. 2015.

Silveira, B. D. da et al.

ISSN eletrônico 1982-4688 / ISSN impresso 0015-3826 
mesmo a ausência de certas espécies nativas da Floresta Estacional Semidecidual, mesmo em áreas isoladas e que não sofrem intervenção humana há décadas.

Nas florestas nativas situadas na região central e na fronteira oeste do Rio Grande do Sul, costuma-se encontrar com certa frequência a espécie Trichilia claussenii C. DC., conhecida popularmente como catiguá. É considerada uma espécie de madeira com resistência e durabilidade moderada, utilizada em acabamentos na construção civil, marcenaria e artesanato. Essa espécie pode ser empregada em adensamento de florestas e na recuperação de áreas degradadas (LORENZI, 2002).

Existe carência de dados sobre muitas espécies nativas, especialmente sobre seu crescimento e potencial produtivo. Estudos com espécies de diferentes utilidades (econômica e/ou ecológica), como, por exemplo, Trichilia claussenii, que não possuía tanta evidência no passado e é considerada de baixo valor econômico, também são necessários para o aprimoramento do conhecimento acerca da formação florestal, e, consequentemente, para sua utilização de forma sustentável e conservação de seus recursos.

O crescimento das árvores é influenciado por fatores genéticos da espécie, que interagem com fatores ambientais, tais como temperatura, precipitação, vento, insolação; características físicas, químicas e biológicas do solo; inclinação, altitude e exposição do terreno, e também com fatores biológicos, como a competição (FINGER, 1992).

A competição talvez seja o fator biológico mais importante no manejo florestal, pois quase todas as intervenções estão relacionadas com a manipulação desse fator ou de condições que o alteram (SCHNEIDER; SCHNEIDER, 2008). Conforme Vanclay (1994), muitos pesquisadores tentaram criar um índice de competição que englobasse os diferentes fatores que o afetam, contudo não obtiveram sucesso em suas tentativas.

Vale salientar que, segundo Assmann (1970), o índice de competição expressa uma estimativa do espaço horizontal ocupado por cada árvore central em relação às suas vizinhas. A maioria desses índices relaciona-se a quatro fatores principais: número de competidores, tamanho e distância das árvores vizinhas e luminosidade (VANCLAY, 1994). Santos et al. (2012), Cunha e Finger (2013) e Weber (2013) são exemplos de pesquisadores que realizaram trabalhos com índices de competição em espécies nativas da flora brasileira, comprovando sua influência no crescimento das diferentes espécies estudadas.

A competição pode ser descrita em função do comprimento e diâmetro de copa, posição sociológica e tendências de crescimento, originando diferentes índices, que também são influenciados, principalmente, pela luz e pelo vento (SCHNEIDER; FINGER, 2000; SCHNEIDER; SCHNEIDER, 2008). A morfometria das árvores e as variáveis dela derivadas permitem entender as relações interdimensionais e reconstruir o espaço ocupado por cada árvore, podendo-se determinar o grau de concorrência a que uma espécie está sujeita dentro de um povoamento, assim como torna-se possível inferir sobre a estabilidade, a vitalidade e a produtividade de cada indivíduo (DURLO; DENARDI, 1998).

Devido à importância da competição no manejo florestal, o presente trabalho teve como objetivo determinar a relação de variáveis morfométricas e de índices de competição com o crescimento em diâmetro de Trichilia claussenii, em um fragmento da Floresta Estacional Semidecidual na região central do Rio Grande do Sul.

\section{MATERIAL E MÉTODOS}

\section{Descrição da área de estudo}

O estudo foi desenvolvido em um fragmento da Floresta Estacional Semidecidual na localidade de Tupancy, município de São Sepé, Rio Grande do Sul. A área total aproximada do remanescente é de 40 hectares e está situada entre as coordenadas $30^{\circ} 12^{\prime} 45^{\prime \prime}$ e $30^{\circ} 13^{\prime} 09^{\prime \prime}$ de latitude sul e $53^{\circ} 42^{\prime} 57^{\prime \prime}$ e $53^{\circ} 42^{\prime} 30^{\prime}$ "de longitude oeste, a aproximadamente 200 metros de altitude. O fragmento está localizado em uma propriedade rural e encontra-se isolado das atividades agropastoris, sem interferência humana há pelo menos 50 anos.

O clima da região, segundo a classificação de Köppen, é do tipo $\mathrm{Cfa}$, subtropical úmido (MORENO, 1961). O solo, de acordo com Scheffer-Basso et al. (2008), pertence à Unidade de Mapeamento Vacacaí e caracteriza-se por possuir textura superficial franco-arenosa no horizonte A e baixo teor de matéria orgânica.

\section{Obtenção dos dados, variáveis mensuradas, processamento e análise dos dados}

Os dados utilizados neste trabalho foram obtidos de indivíduos com diâmetro à altura do peito

(d) igual ou superior a 10,0 centímetros, distribuídos em cinco faixas alocadas aleatoriamente, 
subdivididas em parcelas permanentes de $10 \times 10$ metros (unidades amostrais), totalizando 0,51 hectares. A alocação das faixas acompanhou o gradiente de declividade do terreno, objetivando incluir maior variabilidade florística e fitossociológica. As parcelas foram instaladas no ano de 2011 e remedidas em 2012 e 2013.

Foram amostradas 29 árvores, tentando-se abranger todas as classes diamétricas existentes no fragmento dentro da amplitude apresentada pela espécie, de 10,0 a 33,52 cm de diâmetro, as quais foram denominadas de árvores centrais. $\mathrm{O}$ incremento em diâmetro (id) de cada árvore central foi obtido pela diferença do valor medido na terceira ocasião e na primeira ocasião, dividida por dois.

As concorrentes foram identificadas usando-se um fator de área basal $(\mathrm{k})=1$, conforme a amostragem pontual de Bitterlich (WEST, 2009). Cada árvore central teve as seguintes variáveis mensuradas: altura total e altura comercial do fuste (obtidas com auxílio do hipsômetro Vertex, em metros); CAP (obtida com auxílio de trena graduada em milímetros) e convertida em d (diâmetro à altura do peito); diâmetro da copa (obtido através de mensurações de quatro raios de copa nas direções fixas norte $\left(\mathrm{R} 0^{\circ}\right)$, leste $\left(\mathrm{R} 90^{\circ}\right)$, sul $\left(\mathrm{R} 180^{\circ}\right)$ e oeste $\left(\mathrm{R} 270^{\circ}\right)$, determinados com auxílio de uma bússola e medidos com trena laser, tomando-se como ponto de origem o centro do tronco e distanciando-se até o ponto extremo de projeção da copa (CONDÉ et al., 2013).

$\mathrm{O}$ comprimento de copa foi determinado pela diferença entre altura total e altura do fuste. Foram calculadas mediante relações morfométricas a área de projeção de copa $\left(\mathrm{APC}=(\pi / 4) * \mathrm{dc}^{2}\right)$, a percentagem de copa $(\mathrm{PC}=(\mathrm{cc} / \mathrm{h}) * 100)$, o formal de copa $(\mathrm{FC}=(\mathrm{dc} / \mathrm{cc}))$, o grau de esbeltez $(\mathrm{GE}=\mathrm{h} / \mathrm{d})$, o índice de abrangência $(\mathrm{IA}=\mathrm{dc} / \mathrm{h})$ e o índice de saliência (IS = dc/d) (DURLO; DENARDI, 1998; WINK et al., 2012), conforme ilustra a figura 1.

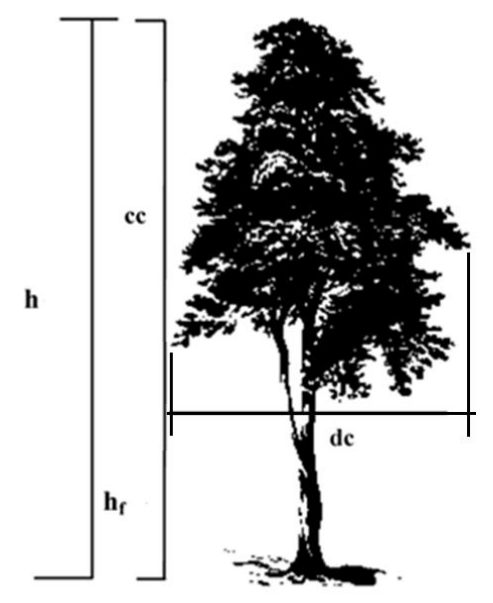

\author{
Parâmetros morfométricos: \\ $\mathrm{d}(\mathrm{cm})$ : diâmetro à altura do peito; \\ $\mathrm{h}(\mathrm{m})$ : altura total da árvore; \\ $\mathrm{h}_{\mathrm{f}}(\mathrm{m})$ : altura de fuste; \\ cc (m): comprimento de copa; \\ dc (m): diâmetro de copa.
}

Figura 1. Representação da altura total e de fuste da árvore, comprimento de copa e diâmetro de copa.

Figure 1. Representation of total height of the tree trunk, crown length and crown diameter.

Fonte: Adaptado de Wink et al. (2012).

Cada árvore central teve as distâncias medidas com trena laser até as árvores concorrentes, consideradas todas as árvores vizinhas com diâmetro mais largo do que a largura da banda $\mathrm{k}=1$ pela amostragem pontual de Bitterlich. As árvores concorrentes tiveram suas alturas totais e diâmetros mensurados, para se quantificar a concorrência de cada árvore, por meio de cálculos dos índices de competição, dependentes e independentes da distância da árvore central à concorrente, como mostra a tabela 1.

Foram usados dois métodos para verificar a existência de relação entre as variáveis morfométricas e os índices de competição com o crescimento das árvores estudadas, quais sejam, índice de correlação de Pearson (r) e análise de regressão por modelagem. Para verificação da existência de correlação pelo índice de Pearson, utilizou-se o índice probabilidade de significância, que varia de 0 a 1 , sendo que quando maior do que 0,05 é não significativo, quando maior que 0,01 e menor ou igual a 0,05 é significativo e quando menor ou igual a 0,01 é altamente significativo.

FLORESTA, Curitiba, PR, v. 45, n. 2, p. 373 - 382, abr. / jun. 2015.

Silveira, B. D. da et al.

ISSN eletrônico 1982-4688 / ISSN impresso 0015-3826 
Tabela 1. Índices de competição utilizados na determinação da concorrência para Trichilia claussenii em um fragmento de Floresta Estacional Semidecidual, São Sepé, RS, 2013.

Table 1. Competition Indices used in determining Trichilia claussenii competition in a Semideciduous Forest fragment, São Sepé, RS, 2013.

\begin{tabular}{|c|c|c|c|}
\hline Índice & Fórmula & Tipo & Fonte \\
\hline $\operatorname{BAL}\left(\mathrm{Bal}_{\mathrm{i}}\right)$ & $\mathrm{Bal}_{i}=\sum_{j=1} G_{j}$ & $\begin{array}{l}\text { Independente da } \\
\text { distância }\end{array}$ & Davis (2001) \\
\hline Glover \& Holl $\left(\mathrm{GH}_{\mathrm{i}}\right)$ & $G H_{i}=d_{i}^{2} / \bar{d}_{j}^{2}$ & $\begin{array}{l}\text { Independente da } \\
\text { distância }\end{array}$ & Husch et al.(2003) \\
\hline $\begin{array}{l}\text { Glover \& Holl } \\
\text { Modificado }\left(\mathrm{GHM}_{\mathrm{i}}\right)\end{array}$ & $G H M_{i}=d_{i}^{2} / \sum_{i=1} \bar{d}_{j}^{2}$ & $\begin{array}{l}\text { Independente da } \\
\text { distância }\end{array}$ & - \\
\hline Hegyi $\left(\mathrm{H}_{\mathrm{i}}\right)$ & $H_{i}=\sum_{j=1}^{n_{j}} \frac{\left(d_{j} / d_{i}\right)}{l_{i j}}$ & $\begin{array}{l}\text { Dependente da } \\
\text { distância }\end{array}$ & Gadow e Hui (1999) \\
\hline $\operatorname{Spurr}\left(\mathrm{G}_{\mathrm{Pi}}\right)$ & $G_{P_{i}}=\frac{k}{n} \sum_{j=1}^{n j}\left[(i-0,5) *\left(\frac{d_{i}}{l_{i j}}\right)^{2}\right]$ & $\begin{array}{l}\text { Dependente da } \\
\text { distância }\end{array}$ & Husch et al. (2003) \\
\hline Spurr modificado $\left(\mathrm{GM}_{\mathrm{Pi}}\right)$ & $G_{P_{i}}=\frac{k}{n} \sum_{j=1}^{n j}\left[(i-0,5) *\left(\frac{\sum_{j=1}^{n j} d_{i}}{l_{i j}}\right)^{2}\right]$ & $\begin{array}{l}\text { Dependente da } \\
\text { distância }\end{array}$ & - \\
\hline $\begin{array}{l}\text { Razão altura/distância } \\
\left(\text { RHL }_{\mathrm{i}}\right)\end{array}$ & $R H L_{i}=\sum^{n j} h_{j} / l_{i j}$ & $\begin{array}{l}\text { Dependente da } \\
\text { distância }\end{array}$ & - \\
\hline
\end{tabular}

n: número de árvores competidoras; $\mathrm{G}_{\mathrm{j}}$ : área basal das árvores competidoras $\left(\mathrm{m}^{2} / \mathrm{ha}\right) ; \mathrm{d}_{\mathrm{i}}$ : dap da árvore central $(\mathrm{cm})$; $\mathrm{d}_{\mathrm{j}}$ : dap das árvores competidoras $(\mathrm{cm}) ; \bar{d}_{j}$ : dap médio das árvores competidoras $(\mathrm{cm}) ; 1_{\mathrm{ijj}}$ : distância entre árvore central i e árvore competidora $\mathrm{j}$ $(\mathrm{m}) ; \mathrm{D}_{\mathrm{C}}$ : diâmetro da copa; $\mathrm{k}$ : fator de área basal; $\mathrm{h}_{\mathrm{j}}$ : altura das árvores competidoras; $\mathrm{i}$ : número de ordem da árvore central considerada; j: número de ordem da árvore competidora.

A modelagem foi realizada com as variáveis independentes originais $\left(\mathrm{x}=\mathrm{BAL}_{\mathrm{i}}, \mathrm{GH}_{\mathrm{i}}, \mathrm{GHM}_{\mathrm{i}}, \mathrm{H}_{\mathrm{i}}\right.$, $\mathrm{GP}_{\mathrm{i}}, \mathrm{RHL}_{\mathrm{i}}$ ) e as transformações de $\mathrm{x}: \mathrm{x}^{2}, \mathrm{x}^{3}, \mathrm{x}^{4}, \mathrm{x}^{5}, 1 / \mathrm{x}, 1 / \mathrm{x}^{2}, 1 / \mathrm{x}^{3}, 1 / \mathrm{x}^{4}, 1 / \mathrm{x}^{5}, \ln \mathrm{x}, \ln \mathrm{x}^{2}, \ln \mathrm{x}^{3}, \ln \mathrm{x}^{4}, \ln \mathrm{x}^{5}$, $\ln 1 / x, \ln 1 / x^{2}, \ln 1 / x^{3}, \ln 1 / x^{4}, \ln 1 / x^{5}$. Foram mantidas, no modelo, as variáveis dependentes com índice de probabilidade até 0,15 , que é o padrão do SAS 9.2, software utilizado nas análises, com a seleção de variáveis pelo método stepwise. No método stepwise, é incluída uma variável dependente após a outra, iniciando com as de coeficiente de determinação $\left(\mathrm{R}^{2}\right)$ parcial mais alto e testando-se a significância de cada novo modelo formado, bem como de cada uma das variáveis.

\section{RESULTADOS E DISCUSSÕES}

Os valores médios, mínimos e máximos das variáveis morfométricas de Trichilia claussenii são apresentados na tabela 2, na qual se observa a heterogeneidade entre os indivíduos amostrados, indicada pela variabilidade dos diâmetros e alturas. As árvores dessa espécie possuem diâmetro pequeno, no entanto podem ser consideradas árvores altas e com copas relativamente pequenas para o fragmento em questão.

O grau de esbeltez, ou seja, a relação altura/diâmetro, pode indicar instabilidade contra o vento, sendo que quanto mais alto, mais instável é a árvore (DURLO; DENARDI, 1998). Valores pequenos indicam que as árvores estão crescendo mais em diâmetro do que em altura (ROVEDA et al., 2013). T. claussenii obteve um grau de esbeltez (GE) variando de 29 a 138. Roman et al. (2009) também afirmam que indivíduos com maiores valores para essa variável são mais instáveis, como o analisado por eles em um estudo com Cordia trichotoma (Vell.) Arráb. ex Steud., em que o grau de esbeltez para essa espécie variou de 47,8 a 130 . Assim, pode-se dizer que T. claussenii é suscetível à ação do vento, como foi observado no campo, quando, após uma tempestade ocorrida em abril de 2012 na região, encontraramse alguns indivíduos dessa espécie caídos, provavelmente derrubados pela ação do vento.

Orellana e Koehler (2008), pesquisando as relações morfométricas de Ocotea odorifera (Vell.) Rohwer em um remanescente da Floresta Ombrófila Mista no Paraná, encontraram um formal de copa (FC) médio de 1,3, variando entre 0,39 e 2,31, sugerindo a existência de uma grande amplitude de variação entre os indivíduos. Os mesmos autores afirmam que valores baixos (menor que 1) indicam árvores com copas 
esbeltas, enquanto valores altos (maiores que 1) indicam copas achatadas. Resultados semelhantes foram encontrados para $T$. claussenii $(\mathrm{FC}=1,56$ ), porém a amplitude entre os valores obtidos foi muito maior no presente estudo (Tabela 2), indicando que essa espécie também pode apresentar uma copa mais achatada do que alongada, sendo esta última o mais comum para sua forma florestal.

Tabela 2. Resultados para as variáveis morfométricas das árvores centrais de Trichilia claussenii em um fragmento de Floresta Estacional Semidecidual, São Sepé, RS, 2013.

Table 2. Results for morphometric variables of Trichilia claussenii central trees in a semideciduous forest fragment, São Sepé, RS, 2013.

\begin{tabular}{lcccc}
\hline Variável & Média & Desvio padrão & Mínimo & Máximo \\
\hline D & 16,19 & 5,38 & 10,19 & 33,52 \\
H & 10,18 & 2,32 & 6,20 & 14,70 \\
CC & 4,82 & 1,95 & 1,60 & 8,90 \\
DC & 6,34 & 1,40 & 4,12 & 9,22 \\
APC & 33,05 & 14,66 & 13,30 & 66,77 \\
PC & 46,22 & 12,50 & 22,67 & 67,12 \\
FC & 1,56 & 0,82 & 0,66 & 4,24 \\
GE & 67,00 & 22,00 & 29,00 & 138,0 \\
IA & 0,65 & 0,20 & 0,34 & 1,17 \\
IS & 0,41 & 0,10 & 0,21 & 0,60 \\
\hline d: diâmetro à altura do peito (cm); h: altura total $(\mathrm{m}) ;$ CC: comprimento de copa (m); DC: \\
diâmetro de copa (m); APC: área de projeção de copa $\left(\mathrm{m}^{2}\right) ;$ PC: percentagem de copa; FC: \\
formal de copa; GE: grau de esbeltez; IA: índice de abrangência; IS: índice de saliência.
\end{tabular}

A relação entre o diâmetro de copa e o diâmetro à altura do peito (d), denominada índice de saliência, foi igual a 0,41 , sugerindo que a espécie estudada possui uma copa de 41 vezes o diâmetro de seu tronco a 1,3 m de altura (d). Segundo Durlo e Denardi (1998), em povoamentos mistos e inequiâneos, essa variável pode ser utilizada para determinar o espaço a ser liberado ao redor de uma árvore selecionada para que ela cresça sem concorrência.

O valor médio para o índice de abrangência, que é explicado pela relação entre o diâmetro de copa e a altura total da árvore, foi de 0,65 para T. claussenii. Durlo e Denardi (1998), estudando a morfometria de Cabralea canjerana (Vell.) Mart., no Rio Grande do Sul, encontraram um valor de 0,33 para o índice de abrangência, afirmando que esse índice pode ser utilizado como indicador da necessidade de intervenções silviculturais, informando o número de árvores que pertencem a um hectare sem sofrer concorrência.

Os resultados médios, mínimos e máximos dos diferentes índices de competição para T. claussenii estão expostos na tabela 3. O índice Bal, definido como a soma das áreas basais das árvores competidoras com área basal maior que a árvore central, demonstra que a espécie sofre alta concorrência de suas vizinhas nesse fragmento florestal, apresentando o valor de 18,57 para esse índice. Cabe aqui salientar que, quanto maior o índice Bal, mais competição sofre a árvore em questão.

Tabela 3. Índices de competição calculados para Trichilia claussenii em um fragmento da Floresta Estacional Semidecidual, São Sepé, RS, 2013.

Table 3. Competition indices calculated for Trichilia claussenii in a semideciduous forest fragment, São Sepé, RS, 2013.

\begin{tabular}{lcccc}
\hline Variável & Média & Desvio padrão & Mínimo & Máximo \\
\hline $\mathrm{Bal}_{\mathrm{i}}$ & 18,57 & 10,77 & 5,20 & 41,74 \\
$\mathrm{GH}_{\mathrm{i}}$ & 0,60 & 0,63 & 0,09 & 2,55 \\
$\mathrm{GHM}_{\mathrm{i}}$ & 0,15 & 0,14 & 0,02 & 0,57 \\
$\mathrm{H}_{\mathrm{i}}$ & 2,24 & 0,84 & 0,48 & 3,92 \\
$\mathrm{G}_{\mathrm{Pi}}$ & 0,31 & 0,13 & 0,09 & 0,66 \\
$\mathrm{GM}_{\mathrm{Pi}}$ & 1,37 & 0,79 & 0,19 & 3,62 \\
$\mathrm{RHL}_{\mathrm{i}}$ & 18,07 & 8,28 & 5,56 & 40,85 \\
\hline
\end{tabular}

Bal ${ }_{\mathrm{i}}$ : índice de $\mathrm{BAL} ; \mathrm{GH}_{\mathrm{i}}$ : índice de Glover \& Holl; $\mathrm{GHM}_{\mathrm{i}}$ : índice de Glover \& Holl modificado; $\mathrm{H}_{\mathrm{i}}$ : índice de Hegyi; $\mathrm{G}_{\mathrm{Pi}}$ índice de Spurr; $\mathrm{GM}_{\mathrm{Pi}}$ índice de Spurr modificado; RHL $\mathrm{i}$ : relação entre altura das árvores competidoras e distância entre a árvore central i e a árvore competidora j; i: número de ordem da árvore central; j: número de ordem da árvore competidora. 
Analisando os resultados da tabela 3 quanto aos índices de competição independentes da distância, Glover \& Hool $\left(\mathrm{GH}_{\mathrm{i}}\right)$ e Glover \& Hool modificado $\left(\mathrm{GHM}_{\mathrm{i}}\right)$, os quais indicam o quanto a área basal da árvore central é maior que a área basal média das concorrentes, verifica-se que $T$. claussenii $\left(\mathrm{GH}_{\mathrm{i}}=0,6\right.$ e $\left.\mathrm{GHM}_{\mathrm{i}}=0,15\right)$ sofre influência da concorrência. Quanto maiores esses índices, mais a árvore central está livre das concorrentes, ou seja, menor é a competição sofrida. Santos et al. (2012), pesquisando Ocotea porosa (Nees) Barroso em um povoamento implantado em 1967, no Paraná, encontraram 1,29 para o índice de Glover \& Hool e afirmam que esse valor ratifica que as árvores dessa espécie estão em alto grau de competição, com seu crescimento influenciado pelas árvores vizinhas.

Em relação aos índices dependentes da distância, o índice de Hegyi $\left(\mathrm{H}_{\mathrm{i}}\right)$ apresentou um valor de 2,24, indicando que sofre grande concorrência das árvores do entorno, confirmando os resultados dos índices independentes da distância. Weber (2013), estudando Ocotea porosa em Santa Catarina, notou que esse índice reflete uma maior competição nas menores classes diamétricas, decrescendo gradativamente até as maiores classes, em que os indivíduos de Ocotea porosa já alcançaram o dossel superior e não se encontram mais em severa concorrência.

Os índices de Spurr $\left(\mathrm{G}_{\mathrm{Pi}}\right)$ e Spurr modificado $\left(\mathrm{GM}_{\mathrm{Pi}}\right)$, assim como a relação altura/distância $\left(\mathrm{RHL}_{\mathrm{i}}\right)$ das árvores competidoras, para T. claussenii, demonstram, assim como os demais, a existência de competição, e quanto maiores esses valores e maior a proximidade das árvores vizinhas, maior será sua influência sobre o crescimento da árvore central.

Os resultados da análise de correlação de Pearson (r) do incremento em diâmetro, variáveis morfométricas e índices de competição para $T$. claussenii são apresentados na tabela 4 , não tendo apresentado valores significativos para $T$. claussenii. No entanto, o crescimento apresentou correlação significativa com o índice Bal $(r=-0,35)$, cujo sinal negativo indica que quanto maior a concorrência, menor é o crescimento. A capacidade preditiva de um índice de competição está muito sujeita à espécie estudada e não à sua complexidade, por isso, em muitos casos, os índices mais simples são mais eficientes (TONINI, 2007), como no caso da T. claussenii, que teve o índice Bal como mais expressivo.

Além da análise de correlação de Pearson, foi realizada análise de regressão por modelagem matemática, procurando-se criar um modelo de equação para descrever o incremento em diâmetro em função dos índices de competição e parâmetros morfométricos. O modelo com as melhores estatísticas para estimar o incremento em diâmetro para $T$. claussenii está apresentado na tabela 5 . A modelagem resultou em um modelo de incremento em diâmetro em função das variáveis índice de saliência e índice Bal, que apresentou um bom ajuste dos dados, levando em consideração o erro padrão da estimativa (Syx $=0,116)$ e as estimativas para as estatísticas de coeficiente de determinação $\left(R^{2}=0,715\right)$, conforme exposto na tabela 5 .

Esses resultados confirmam os obtidos na tabela 4, os quais mostram que o índice Bal foi significativo pela análise de correlação de Pearson. O modelo obtido indica, através do índice Bal e do índice de saliência, a influência do espaço livre em torno de cada árvore central, apontando que T. claussenii cresce mais sem concorrência e pode ter um acréscimo significativo em área basal e, portanto, em volume por hectare, se for cultivada com espaçamento adequado.

Weber (2013) também incluiu em seu modelo de incremento em área basal para Ocotea porosa, em Santa Catarina, os índices de saliência e de competição Bal, justificando a inserção dessas variáveis em função de a espécie ser extremamente exigente em luminosidade. Essa mesma explicação pode ser usada para o modelo obtido para T. claussenii, que, conforme Lorenzi (2002), é uma planta esciófita ou de luz difusa, isto é, tolera a sombra nas etapas iniciais de seu desenvolvimento, contudo demanda um alto grau de iluminação para passar pelas etapas intermediárias até a maturidade, aumentando o seu crescimento se ocorrer uma abertura no dossel (MACIEL et al., 2003).

A relação entre incremento em diâmetro e diâmetro à altura do peito possui uma dispersão significativa entre os valores observados, expondo a variação de diâmetros na floresta, indicando que mesmo indivíduos com diâmetros maiores não apresentam, necessariamente, maior crescimento em diâmetro, o que pode ser justificado pela competição sofrida. Os indivíduos com copas maiores e menor competição têm maior incremento em diâmetro, de modo que há indivíduos de maior diâmetro, mas pequeno incremento diamétrico e vice-versa, causando a grande dispersão dos pontos em todas as classes diamétricas no gráfico.

O estudo da morfometria e da competição é extremamente importante para auxiliar no conhecimento do crescimento de diferentes espécies florestais e, principalmente, no seu manejo, o que se 
verifica também neste trabalho. Segundo Curto et al. (2013), para a realização do manejo florestal, visando à produção sustentável, torna-se de fundamental relevância conhecer as características da floresta. Assim, os resultados obtidos nesta pesquisa demonstram que existe influência da competição no incremento em diâmetro de T. claussenii, com cada indivíduo reagindo ao espaço e à competição, o que explica o crescimento diamétrico diferenciado de uma árvore para outra com a mesma dimensão do tronco.

Tabela 4. Coeficientes de correlação de Pearson entre o incremento em diâmetro e as variáveis morfométricas e os índices de competição para Trichilia claussenii em um fragmento de Floresta Estacional Semidecidual, São Sepé, RS, 2013.

Table 4. Pearson correlation coefficients between diameter increment and morphometric variables and the competition indexes for Trichilia claussenii in a semideciduous forest fragment, São Sepé, RS, 2013.

\begin{tabular}{lc}
\hline Variáveis & $\mathbf{r}$ \\
\hline $\mathrm{D}$ & $-0,066^{\mathrm{ns}}$ \\
$\mathrm{H}$ & $0,0575^{\mathrm{ns}}$ \\
$\mathrm{CC}$ & $0,1562^{\mathrm{ns}}$ \\
$\mathrm{DC}$ & $0,2167^{\mathrm{ns}}$ \\
$\mathrm{APC}$ & $0,2024^{\mathrm{ns}}$ \\
$\mathrm{PC}$ & $0,1414^{\mathrm{ns}}$ \\
$\mathrm{FC}$ & $-0,0853^{\mathrm{ns}}$ \\
$\mathrm{GE}$ & $0,0307^{\mathrm{ns}}$ \\
$\mathrm{IA}$ & $0,0444^{\mathrm{ns}}$ \\
$\mathrm{IS}$ & $0,2412^{\mathrm{ns}}$ \\
$\mathrm{G}_{\mathrm{Pi}}$ & $-0,2202^{\mathrm{ns}}$ \\
$\mathrm{GM}$ & $0,0313^{\mathrm{ns}}$ \\
$\mathrm{BAL}$ & $-0,3591^{*}$ \\
$\mathrm{H}_{\mathrm{i}}$ & $-0,1927^{\mathrm{ns}}$ \\
$\mathrm{GH}_{\mathrm{i}}$ & $-0,0164^{\mathrm{ns}}$ \\
$\mathrm{GHM}_{\mathrm{i}}$ & $0,0253^{\mathrm{ns}}$ \\
$\mathrm{RHL}_{\mathrm{i}}$ & $-0,1630^{\mathrm{ns}}$ \\
\hline
\end{tabular}

r: coeficiente de correlação linear de Pearson; d: diâmetro das árvores centrais; h: altura total das árvores centrais; CC: comprimento de copa; DC: diâmetro de copa; APC: área de projeção de copa; PC: percentagem de copa; FC: formal de copa; GE: grau de esbeltez; IA: índice de abrangência; IS: índice de saliência; $\mathrm{G}_{\mathrm{pi}}$ : índice de Spurr; $\mathrm{GM}_{\mathrm{Pi}}$ : índice de Spurr modificado; $\mathrm{Bal}_{\mathrm{i}}$ : índice basal area larger; $\mathrm{H}_{\mathrm{i}}$ : índice de Hegyi; $\mathrm{GH}_{\mathrm{i}}$ : índice de Glover \& Holl; $\mathrm{GHM}_{\mathrm{i}}$ : índice de Glover \& Holl modificado; RHL $_{\mathrm{i}}$ : relação entre altura das árvores competidoras e distância entre a árvore central i e a árvore competidora $\mathrm{j}$; ${ }^{\text {ns }}$ : valor de $\mathrm{r}$ com probabilidade não significativa; ${ }^{*}$ : valores de r significativos a $5 \%$ de probabilidade.

Tabela 5. Modelo matemático para estimar incremento em diâmetro (id) em função dos índices de saliência (IS) e competição (BAL), com seus respectivos coeficiente de determinação $\left(\mathrm{R}^{2}\right)$, coeficiente de determinação ajustado $\left(\mathrm{R}_{\text {aj. }}^{2}\right.$ ) e erro padrão da estimativa (Syx) para Trichilia claussenii em um fragmento de Floresta Estacional Semidecidual, São Sepé, RS, 2013.

Table 5. A mathematical model to estimate diameter increment (id), with their respective determination coefficient $\left(\mathrm{R}^{2}\right)$, adjusted determination coefficient $\left(\mathrm{R}_{\mathrm{aj} .}\right.$ ) and standard error of estimate (Syx) for Trichilia claussenii in a semideciduous forest fragment, São Sepé, RS, 2013.

\begin{tabular}{lccc}
\hline Modelo & $\mathbf{R}^{\mathbf{2}}$ & $\mathbf{R}_{\text {aj. }}^{2}$ & $\mathbf{S y x}$ \\
\hline$i d=0,4845 * I S-0,0018 *$ Bal & 0,7150 & 0,6947 & 0,1161 \\
\hline IS: índice de saliência; Bal: índice basal area larger. & & &
\end{tabular}

Além dos parâmetros aqui apresentados, todos os outros fatores genéticos, biológicos e ambientais influenciam no crescimento das árvores, necessitando de estudos mais aprofundados. Vale ressaltar que $T$. claussenii possui potencial para ser utilizada como espécie comercial, desde que seja avaliada e melhorada geneticamente, assim como muitas outras espécies da vasta flora brasileira. Portanto, este estudo confirma um pouco da complexidade do entendimento das florestas, especialmente 
das nativas, mostrando a verdadeira necessidade de pesquisas nessa área que possam fornecer os dados necessários para sua conservação.

\section{CONCLUSÃO}

- Existe associação entre o incremento em diâmetro de Trichilia claussenii e a competição, em que o crescimento em diâmetro possui maior relação com o índice de competição Bal. Quanto maior a competição, menor o crescimento, mostrando que a espécie é fortemente afetada pelas competidoras.

- A relação do crescimento com o índice de saliência (IS) apontou que, quanto maior a copa, maior o crescimento da espécie. Ou seja, árvores de T. claussenii com copa grande e pouca competição crescem mais, inferindo-se que o ajuste da densidade por meio de intervenções silviculturais pode resultar em maior crescimento, constituindo-se em informação importante para o manejo florestal.

\section{REFERÊNCIAS}

ASSMAN, E. The Principles of Forest the Yield Study. München: BLV, 1970. 490 p.

CONDÉ, T. M.; LIMA, M. L. M.; LIMA NETO, E. M.; TONINI, H. Morfometria de quatro espécies florestais em sistemas agroflorestais no munícipio de Porto Velho, Rondônia. Revista Agro@mbiente On-line, Boa Vista, v. 7, n. 1, p. 18-27, 2013. Disponível em: <http://revista.ufrr.br/index.php/ agroambiente/article/viewFile/932/1016>. Acesso em: 31/05/2014.

CUNHA, T. A.; FINGER, C. A. G. Competição assimétrica e o incremento diamétrico de árvores individuais de Cedrela odorata L. na Amazônia Ocidental. Acta Amazonica, Manaus, v. 43, n. 1, p. 9 $18,2013$.

CURTO, R. de A.; SILVA, G. F. da; SOARES, C. P. B.; MARTINS, L. T.; DAVID, H. C. Métodos de estimação de altura de árvores em Floresta Estacional Semidecidual. Floresta, Curitiba, v. 43, n. 1, p. 105 - 116, 2013.

DAVIS, L. S.; JOHNSON, K. M.; BETTINGER, P.; HOWARD, T. Forest Management: to Sustain Ecological, Economic and Social Values. New York: Macgrall-Hill, 2001. 4th. ed. 816 p.

DURLO, M. A.; DENARDI, L. Morfometria de Cabralea canjerana, em mata secundária nativa do Rio Grande do Sul. Ciência Florestal, Santa Maria, v. 8, n. 1, p. 55 - 66, 1998.

FINGER, C. A. G. Fundamentos de Biometria Florestal. Santa Maria: UFSM/CEPEF/FATEC, 1992. $269 \mathrm{p}$.

GADOW, K. V.; HUI, G. Modelling Forest Development. Dordrecht: Kluwer Academic Publishers, 1999, 213 p.

HUSCH, B.; BEERS, T. W; KERSHAW, J. A. Forest Mensuration. New Jersey: John Wiley \& Sons Inc., 2003, 4th. ed., 456 p.

LONGHI, S. J.; ARAÚJO, M. M.; KELlinG, M. B.; HOPPE, J. M.; MÜLLER, I.; BORSOI, G. A. Aspectos fitossociológicos de fragmento de Floresta Estacional Decidual, Santa Maria, RS. Ciência Florestal, Santa Maria, v. 10, n. 2, p. 59 - 74, 2000.

LORENZI, H. Árvores Brasileiras: manual de identificação e cultivo de plantas arbóreas nativas do Brasil. Nova Odessa, SP: Instituto Plantarum. v. 2, 2. ed., 2002. 368 p.

MACIEL, M. de N. M.; WATZLAWICK, L. F.; SCHOENINGER, E. R.; YAMAJI, F. M. Classificação ecológica das espécies arbóreas. Revista Acadêmica: ciências agrárias e ambientais, Curitiba, v. 1, n. 2, p. 69 - 78, 2003.

MORENO, J. A. O clima do Rio Grande do Sul. Porto Alegre: Secretaria da Agricultura, 1961. 41 p.

ORELlANA, E.; KOEHLER, A. B. Relações morfométricas de Ocotea odorifera (Vell.) Rohwer. Revista Acadêmica Ciências Agrárias e Ambientais, Curitiba, v. 6, n. 2, p. 229 - 237, 2008. 
ROMAN, M.; BRESSAN, D. A.; DURLO, M. A. Variáveis morfométricas e relações interdimensionais para Cordia trichotoma (Vell.) Arráb. ex Steud. Ciência Florestal, Santa Maria, v. 19, n. 4, p. 473 - 480, 2009.

ROVEDA, M.; DALGAllo, B.; DIAS, A. N.; FIGUEIREDO FILHO, A.; MULleR, C. S. Morfometria de Araucaria angustifolia (Bertol.) Kuntze na Floresta Nacional de São Francisco de Paula, RS. Disponível em: <http://ebookbrowsee.net/sil-artigo-38-pdf-d456146397>. Acesso em: 03/07/2013.

SANTOS, A. T.; MATTOS, P. P.; BRAZ, E. M.; ROSOT, N. C. Índices de competição em plantio de Ocotea porosa. Comunicado Técnico 295, Embrapa Florestas, Colombo, p. 1 - 5, 2012.

SCHEFFER-BASSO, S. M.; SCHERER, C. V.; ELLWANGER, M. F. Resposta de pastagens perenes à adubação com chorume suíno: pastagem natural. Revista Brasileira de Zootecnia, Viçosa, v. 37, n. 2, p. $221-227,2008$.

SCHNEIDER, P. R.; FINGER, C. A. G. Manejo sustentado de florestas inequiâneas heterogêneas. Santa Maria: UFSM, 2000, 195 p.

SCHNEIDER, P. R.; SCHNEIDER, P. S. P. Introdução ao manejo florestal. Santa Maria: FACOS/UFSM, 2. ed., 2008, 566 p.

TONINI, H. Índice de competição e o seu uso na modelagem do crescimento das árvores. Boa Vista: Embrapa Roraima (Documentos / Embrapa Roraima; 8), 2007. 30 p.

VANCLAY, J. K. Modeling forest growth and yield: aplications to mixed tropical forests. Copenhagen: CAB International, 1994, 312 p.

WEBER, V. P. Relação morfométrica e de competição no crescimento de Ocotea porosa (Nees et Martius ex Nees) Liberato Barroso na Região Central de Santa Catarina. $91 \mathrm{f}$. Tese (Doutorado em Engenharia Florestal) - Universidade Federal de Santa Maria, Santa Maria, 2013.

WEST, P. W. Tree and forest measurement. Berlim, 2. ed., 2009, 190 p.

WINK, C.; MONTEIRO, J. S.; REINERT, D.; LIBERALESSO, E. Parâmetros da copa e a sua relação com o diâmetro e altura das árvores de eucalipto em diferentes idades. Scientia Forestalis, Piracicaba, v. 40, n. 93 , p. $57-67,2012$. 
FLORESTA, Curitiba, PR, v. 45, n. 2, p. 373 - 382, abr. / jun. 2015. Silveira, B. D. da et al. 